\title{
Results of Surgical Treatment of Patients with Critical Limb Ischemia and Stenotic Lesions of the Brachiocephalic Arteries
}

\author{
Alexei L. Charyshkin, $\mathrm{PhD}, \mathrm{ScD}^{1 *}$; Alexander A. Maksin, $\mathrm{PhD}^{2}$; \\ Lyubov V. Matveeva, $\mathrm{PhD}^{1}$; Alexander V. Poseryaev ${ }^{2}$; Maksim V. Yashkov ${ }^{1}$ \\ ${ }^{I}$ Institute of Medicine, Ecology and Physical Education of Ulyanovsk State University \\ ${ }^{2}$ Ulyanovsk Regional Hospital \\ Ulyanovsk, The Russian Federation
}

\begin{abstract}
The aim of our study was to evaluate the results of the surgical treatment for patients with critical limb ischemia (CLI) and stenotic lesions of the brachiocephalic arteries.

Methods and Results: We examined 72 patients (68/87.2\% men and 4/7.3\% women) aged from 46 to 78 years (mean age, $62.2 \pm 4.3$ years) with CLI and stenotic lesions of the brachiocephalic arteries. Conservative treatment was performed in $17(23.6 \%)$ patients and surgical treatment in 55(76.4\%). It has been carried out 73 surgical operations: femoral popliteal bypass $(5 / 6.8 \%)$, lumbar sympathectomy (4/5.5\%), thrombectomy of occluded aortofemoral graft (2/2.7\%), limb amputation (4/5.5\%), iliofemoral bypass (4/5.5\%), aortofemoral bifurcation bypass (10/13.1\%), endovascular surgery (1/1.6\%), limb amputation at thigh level $4(5.5 \%)$, thrombectomy of occluded distal arteries $(4 / 5.5 \%)$, femoro-femoral cross-over bypass $(1 / 1.6 \%)$, resection of popliteal artery aneurysm and prosthesis of the popliteal artery $(1 / 1.6 \%)$, semi-closed loop endarterectomy of occluded arteries of the lower limbs (8/10.9\%), carotid endarterectomy (23/31.5\%), and carotid-subclavian bypass (2/2.7\%). After the surgical intervention, we observed the disappearance or reduction of pain, restoration of sensitivity and motor activity, and healing of trophic ulcers in $75 \%$ of patients. In the late postoperative period, we detected the progression of limb ischemia in 4(5.5\%) patients; in connection with that, we performed limb amputation at thigh level. Ischemic stroke with a lethal outcome developed in one patient (1.4\%).

Conclusion: In patients with multifocal atherosclerosis, multilevel reconstructive surgical interventions must be performed in stages, due to the high operational risk, and risk of complications, secondary amputations and lethality in the postoperative period. (International Journal of Biomedicine. 2017;7(2):147-149.)
\end{abstract}

Key Words: critical limb ischemia $\bullet$ multifocal atherosclerosis $\bullet$ postoperative complications $\bullet$ concomitant diseases

\section{Abbreviations}

BAs, brachiocephalic arteries; CLI, critical limb ischemia; MFA, multifocal atherosclerosis; PAD, peripheral artery disease.

\section{Introduction}

Critical limb ischemia (CLI), which is at the end of the peripheral artery disease (PAD) spectrum, is associated with excessively high risk for cardiovascular events, including

*Corresponding author: Prof. Alexei L. Charyshkin, PhD, ScD, Head of the Faculty Surgery Department, Institute of Medicine, Ecology and Physical Education, Ulyanovsk State University. Ulyanovsk, The Russian Federation.E-mail: charyshkin@yandex.ru myocardial infarction, and death..$^{(1,2)}$ CLI with multifocal atherosclerotic lesions and concomitant diseases is an urgent problem of vascular surgery. ${ }^{(3-6)}$ Recently, the number of patients with MFA and CLI has increased, largely the result of an increase in the number of elderly patients. ${ }^{(3,6-9)}$ According to the literature, CLI complicates the course of eliminating atherosclerosis of the lower limbs in $33 \%$ of patients. ${ }^{(6,10-12)}$ As a result, the lethality and number of amputations now reaches $14.0 \%$ and $20.4 \%$, respectively, in CLI patients after reconstructive interventions on the vascular bed..$^{(5-7,13)}$ 
Reconstructive surgical interventions provide preservation of limbs in the postoperative period in terms up to 5 years in $80 \%$ of patients, up to 10 years - only in $50 \%$. $^{(4-7)}$

To this day, the choice of the optimal treatment option for patients with CLI and MFA remains one of the most difficult and controversial problems for representatives of various surgical schools and directions due to the high risk of complications with reconstructive interventions, which is due to the age of patients and the severity of the concomitant pathology.

The aim of our study was to evaluate the results of the surgical treatment for patients with CLI and stenotic lesions of the brachiocephalic arteries (BAs).

\section{Materials and Methods}

We examined 72 patients $(68 / 87.2 \%$ men and $4 / 7.3 \%$ women) aged from 46 to 78 years (mean age, 62.2 \pm 4.3 years) with CLI and stenotic lesions of BAs who underwent surgical treatment in the department of vascular surgery of the Regional Oncology Center. Inclusion criteria were patients with lower limb ischemia (Fontaine III or IV) of atherosclerotic genesis in combination with stenotic lesions of BAs, who have had the disease for at least three years and are undergoing in-patient treatment courses at least twice a year. Diagnostic tests included ultrasound duplex scanning of the vessels, assesment of tissue blood flow in the distal parts of limbs using laser Doppler flowmetry, and aortic angiography. The indications for surgery were the decompensated stage of peripheral circulation, ineffective conservative therapy, and angiographic signs of the presence of blood flow in the deep femoral artery, its branches, the distal portion of the popliteal artery, and at least one of the tibial arteries. The decision to conduct operative intervention was strictly individual, taking into account the patient's condition, the nature and degree of arterial damage, the state of the distal bed, the stage of the disease, and the presence and severity of the concomitant pathology. Contraindications were the total occlusion of the deep and superficial femoral arteries, occlusions of the popliteal, anterior and posterior tibial arteries, severe limb ischemia with the presence of tension and contraction of the calf muscle, and the presence of wet gangrene. The choice of the method of reconstructive surgery was determined by the level of damage, the prevalence of the obliterating process, and the severity of the concomitant pathology.

Conservative treatment was performed in $17(23.6 \%)$ patients and surgical treatment in 55(76.4\%). It has been carried out 73 surgical operations: femoral popliteal bypass $(5 / 6.8 \%)$, lumbar sympathectomy $(4 / 5.5 \%)$, thrombectomy of occluded aortofemoral graft $(2 / 2.7 \%)$, limb amputation (4/5.5\%), iliofemoral bypass (4/5.5\%), aortofemoral bifurcation bypass $(10 / 13.1 \%)$, endovascular surgery $(1 / 1.6 \%)$, limb amputation at thigh level - 4(5.5\%), thrombectomy of occluded distal arteries (4/5.5\%), femoro-femoral cross-over bypass ( $1 / 1.6 \%)$, resection of popliteal artery aneurysm and prosthesis of the popliteal artery (1/1.6\%), semi-closed loop endarterectomy of occluded arteries of the lower limbs (8/10.9\%), carotid endarterectomy (23/31.5\%), and carotid-subclavian bypass (2/2.7\%).
The study was conducted in accordance with ethical principles of the Declaration of Helsinki. Written informed consent was obtained from all participants.

In the postoperative period, all patients received anticoagulant and disaggregating therapy. Statistical analysis was performed using the statistical software «Statistica» (v6.0, StatSoft, USA).

\section{Results and Discussion}

Given the etiological factors, the following nosological forms of PAD were identified: atherosclerosis $(70 / 97.2 \%)$, Buerger's disease (1/1.4\%), and diabetes mellitus (1/1.4\%). Critical ischemia of the lower limbs was detected in $70(97.3 \%)$, upper limbs in 2(2.7\%) patients. Hemodynamically insignificant and significant stenoses of BAs were detected in $47(65.2 \%)$ and $25(34.8 \%)$ patients, respectively. Trophic changes in distal sections of the lower limbs were observed in 53(73.6\%) patients. Ischemic rest pain was in $19(26.4 \%)$ patients. Angiographic and ultrasound investigations of arterial pools revealed that all CLI patients had multifocal vessel lesions, namely, lesions of the arteries of the lower limbs and BAs. Trophic lesions and clinical manifestations were determined by the level and amount of obliterated arteries.

In patients with critical stenosis of BAs and arteries of the lower limbs, surgical interventions were performed in stages (with a minimum interval between stages): first on the major arteries of the head, then on the arteries of the limbs. The primary restoration of blood flow in BAs was performed to reduce the risk of fatal complications in CLI patients. The main clinical criteria for the effectiveness of treatment were the preservation of the functioning limb, epithelization of ulcers, and the absence of rest pain and neurological symptoms.

After the surgical intervention, we observed the disappearance or reduction of pain, restoration of sensitivity and motor activity, and healing of trophic ulcers in $75 \%$ of patients. In CLI patients, the risk of amputation of the affected limb and a lethal outcome during the first year after surgery is extremely high. In the late postoperative period, we detected the progression of limb ischemia in $4(5.5 \%)$ patients; in connection with that, we performed limb amputation at thigh level. Ischemic stroke with a lethal outcome developed in one patient (1.4\%). Prognosis after surgery in elderly patients with CLI is determined by the type, level and volume of the vascular reconstruction, the initial degree of limb ischemia and the presence and severity of concomitant pathologies, as well as the severity and duration of the recovery period. All of the above have a direct impact on the quality of life of geriatric patients and the results of reconstructive surgery. $(6,13)$

Very important is not only the careful selection of patients with a comprehensive assessment of the reserve and compensatory capabilities of the body, but also the targeted preoperative preparation and prevention of cardiac, pulmonary and other complications, and wide involvement of specialists in related fields that can reduce the risk of postoperative complications and lethality. ${ }^{(6,7,13)}$

Thus, surgical treatment of CLI patients with MFA is a complex task due to the multilevel lesion of the vascular bed 
and severe concomitant diseases. Using a combination of open and endovascular methods to restore blood flow, an individual approach to the choice of surgical method, and adequate medication support leads to success in preserving the limbs and improving the quality of life of these patients.

\section{In conclusion,}

- with MFA, multilevel reconstructive surgical interventions must be performed in stages, due to the high operational risk, and risk of complications, secondary amputations and lethality in the postoperative period;

- complex surgical treatment of patients with CLI and stenotic lesions of BA with the minimal time interval between stages allows reducing the number of amputation of the lower limbs to $5.5 \%$ and lethality to $1.4 \%$.

\section{Competing Interests} interests.

The authors declare that they have no competing

\section{Sources of Funding}

This study was funded by the Ministry of Education and Science of the Russian Federation (the science project No. 18.7236.2017/BCh).

\section{References}

1. Murabito JM, Evans JC, Nieto K, Larson MG, Levy D, Wilson PW. Prevalence and clinical correlates of peripheral arterial disease in the Framingham Offspring Study. Am Heart J. 2002;143:961-965.

2. Teraa M, Conte MS, Moll FL, Verhaar MC. Critical Limb Ischemia: Current Trends and Future Directions. J Am Heart Assoc. 2016;5(2). pii: e002938. doi: 10.1161/
JAHA.115.002938.

3. Abalmasov KG, Buziashvili II, Morozov KM. [Quality of life of patients with chronic ischemia of the lower limbs]. Angology and Vascular Surgery (Moscow). 2004; 10(2):8-13. [Article in Russian].

4. Gavrilenko AV. [Assessment of quality of life in patients with critical ischemia of the lower limbs]. Angology and Vascular Surgery (Moscow). 2001;7(3):8-14. [Article in Russian].

5. Galstyan GE. [Algorithm for diagnosis and treatment of the peripheral artery disease]. Consilium medicum. 2006;8(12):34-8.[Article in Russian].

6. Katelnitsky II. [Opportunities of operative treatment of geriatric patients with critical ischemia of the lower limbs]. Uspekhi gerontologii. 2012;25(3):338-42. [Article in Russian]. 7. Shevchenko YuL. [Medico-biological and physiological basis of cellular technologies in cardiovascular surgery]. SPb.: Nauka; 2006. [in Russian].

8. Dominguez LJ, Barbagallo M, Sowers JR, Resnick LM. Magnesium responsiveness to insulin and insulin-like growth factor I in erythrocytes from normotensive and hypertensive subjects. J Clin Endocr Metab. 1998; 83(12):4402-7.

9. Ruth SoRelle. Dilemma of Angiogenesis. Circulation. 2000;101:e23-e24

10. Rutherford RB, Baker JD, Ernst C, Johnston KW, Porter JM, Ahn S, et al. Recommended standards for reports dealing with lower extremity ischemia: revised version. Vasc Surg. 1997;26(3):516-38.

11. Sandberg T, Ehinger A, Casslén B. Paracrine stimulation of capillary cell migration tissue involves epidermal growth factor and is mediated viaurokinase plasminogen activator receptor. J Clin Endocr Metab.2001;86(4):1724-30.

12. Shintani S, Murohara T, Ikeda H, Ueno T, Sasaki K, Duan J, Imaizumi T. Augmentation of postnatal neovascularization with autologous bone marrow transplantation. Circulation. 2001;103(6):897-903.

13. Simons M. Angiogenesis: where do we stand now? Circulation. 2005;111(12):1556-66. 